\title{
Watermarking via zero assigned filter banks
}

\author{
Zeynep Yücel * , A. Bülent Özgüler \\ Electrical and Electronics Engineering Department, Bilkent University, 06800 Bilkent, Ankara, Turkey
}

\section{A R T I C L E I N F O}

\section{Article history:}

Received 28 September 2008

Received in revised form

20 February 2009

Accepted 6 July 2009

Available online 17 July 2009

\section{Keywords:}

Zero assignment

Wavelets

Watermarking

\begin{abstract}
A B S T R A C T
In order to identify the owner and distributor of digital data, a watermarking scheme in frequency domain for multimedia files is proposed. The scheme satisfies the imperceptibility and persistence requirements and it is robust against additive noise. It consists of a few stages of wavelet decomposition of several subblocks of the original signal using special zero assigned filter banks. By assigning zeros to filters on the high frequency portion of the spectrum, filter banks with frequency selective response are obtained. The information is then inserted in the wavelet-decomposed and compressed signal. Several robustness tests are performed on male voice, female voice, and music files, color and gray level images. The algorithm is tested under white Gaussian noise and against JPEG compression and it is observed to be robust even when exposed to high levels of corruption.
\end{abstract}

(c) 2009 Elsevier B.V. All rights reserved.

\section{Introduction}

Due to the recent developments in Internet and multimedia services, digital data have become easily attainable through the World Wide Web. Properties such as error-free reproduction, efficient processing and storage, and a uniform format for digital applications, make digital technology popular. However, these advantages may present many complications for the owner of the multimedia data. Unrestricted access to intellectual property and the ease of copying digital files raise the problem of copyright protection.

In order to approve rightful ownership and prevent unauthorized copying and distribution of multimedia data, digital watermarking is employed and imperceptible data are embedded into digital media files. Watermarking makes it possible not only to identify the owner or distributor of digital files but also to track the creation or manipulation of audio, image or video signals. Moreover, by embedding a digital signature, one may provide

\footnotetext{
* Corresponding author.

E-mail addresses: zeynep@ee.bilkent.edu.tr (Z. Yücel), ozguler@ee.bilkent.edu.tr (A.B. Özgüler).
}

different access levels to different users. There are several essential conditions that must be met by an effective watermarking algorithm.

(i) The signature of the author, the watermark, needs to be not only transparent to the user but also robust against attacks [8]. These attacks may include degradations resulting from a transmission channel, compression of the signal, rotation, filtering, permutations or quantization.

(ii) The watermarking procedure should be invertible. The watermark must be recovered from the marked data preferably without accessing the original signal.

(iii) The marking procedure must be able to resolve rightful ownership when multiple ownership claims are made. A pirate may modify the marked signal in a way that if his fake original signal is used in detection process, both claimers may gather equal evidence for ownership [13]. This situation is called the deadlock problem [8]. The importance of decoding without the original signal arises here.

(iv) The author should provide secret keys in order to obtain a more secure encryption technique that allows only the authorized detections of the water- 
mark with the help of proper keys. Even if the exact algorithm is available to a pirate, he should not be able to extract or predict the watermark without accessing the security keys.

Since human auditory system (HAS) and human visual system (HVS) are imperfect detectors, the watermark can be made imperceptible via appropriate masking. In visual masking, watermark signal is usually embedded in the detail bands of the signal, which may make the watermark more fragile against certain attacks such as high frequency filtering. Imperceptibility should be counterbalanced against robustness. Wavelets and filter banks offer a great deal of advantage in terms of these requirements.

Watermarking may be performed in spatial domain or in frequency domain. In this paper we deal with watermarking in frequency domain via wavelets and zero assigned filter banks.

Previous works in frequency domain watermarking are addressed in $[4,8,20]$. Wang et al. discuss the practical requirements for watermarking systems [20]. For standardized algorithms storing watermarks, original or marked signals and secret keys may introduce excessive memory requirements and a great deal of financial burden for registration of all those by the legal authority. A good marking scheme should meet several requirements as explained in detail by Swanson et al. [8]. Embedding data must not violate the perceptual quality of the host signal. The mark should be easily detectable. It is also a desired property that the recovery of data does not use the original signal to decode the embedded watermark. Furthermore it must be robust against modifications and manipulations such as compression, filtering, and additive noise.

One of the early image watermarking techniques using wavelets was suggested by Xia et al. [21] (also see [7]), where a white noise with masking was added on top of the detail portions, i.e., High-Low $(H L)$, Low-High $(L H)$ or High-High $(\mathrm{HH})$ bands of the discrete wavelet transform of the image. Since compression schemes degrade the $\mathrm{HH}$ band most, $L H$ or $H L$ band is preferred to obtain robustness against compression. The detection scheme of [21] consisted of computing the correlation of the extracted watermark with the original watermark signal so that one needs to store the embedded watermark and transmit it to the decoder side. Embedded zero-tree wavelets (EZW) have also been employed in watermarking applications for selecting the appropriate detail band coefficients for embedding the watermark [4,15]. In 1993, Shapiro proposed an efficient low bit rate image coding algorithm based on the self-similarity of wavelet coefficients [14]. He found out that if the coefficients at a coarser scale are insignificant with respect to some amplitude threshold $T$, then the ones which correspond to the same spatial location at a finer scale are also likely to be insignificant with respect to $T$. A coefficient at a coarse scale satisfying this self-similarity condition is called the parent and the coefficients corresponding to the same spatial location at finer scales are called its children. Identifying the parents and their children which are insignificant with respect to $T$, one constructs a zero tree which helps in the detection of the perceptually inconsequential regions and embeds a signature there. Because of the spread spectrum handling of data offered by the multiresolution property of the filter banks, there is an opportunity to increase the robustness while keeping the degradations as small as possible [4]. In [15], in order to facilitate the decoding phase of the watermark, rather than erasing the insignificant coefficients, a nonzero number called the embedded intensity replaces these coefficients. In the decoding phase, the mean of the coefficients which are known to be on the zero tree is computed and the correct embedded bit is determined according to the sign of the mean value. In [6], another method based on the idea of EZW is proposed based on 'qualified significant coefficients' that are between two thresholds $T_{1}$ and $T_{2}$. In [10], Mihçak et al. develop an algorithm based on deriving robust semi-global features in wavelet domain and quantizing them. They partition the DC subband into nonoverlapping rectangles and form a series composed of the averages of these rectangles. The watermark embedding is done byquantization of this series. Two different quantization functions are used in order to differentiate between the embedded bits. The authors state that this method is robust against several benchmark attacks and compression.

In [3], Tekalp et al. propose an alternative algorithm to solve the deadlock problem. The authors assume that the number of users of secret files are not many and they can embed a unique watermark into each file composed of a pseudonoise pattern which defines a particular user. Against collusion attacks, the authors propose to apply pre-warping on the host signal. In case of a collusion attack, the method ensures that there will be a perceptual degradation on the signal and the attack will be obvious.

Initial results on the watermarking method given here has been reported in [23,24]. We then adapted the zeroassigned filter bank method of [23] to image watermarking while making the method more robust against attacks via employment of embedded zero-tree wavelets. While keeping the advantages of the earlier image watermarking techniques, we reduce the amount of key information needed in the detection phase. The proposed scheme embeds the watermark in the perceptually transparent parts of the image by replacing the zero-tree elements by an embedded intensity, $+m$ or $-m$, which leads to a reduction in the amount of transmitted information. When the watermark is regarded as a noise introduced on the image, peak signal-to-noise ratio (PSNR) is a good measure of imperceptibility and it must be as high as possible. On the other hand, successful detection of the watermark under as low a PSNR as possible is a measure of the robustness of the method against distortion type of attacks. We illustrate in detail under white Gaussian noise and compression attacks that the method we propose improves both PSNR properties in comparison to the earlier techniques proposed in [11,12,2]

The outline of this paper is as follows. The design algorithm of perfect reconstruction (PR) zero assigned filter banks is discussed in Section 2. In Section 3, the application of zero assigned filter banks in audio and 
image watermarking is explained in detail and several experimental results in noise free, noisy and attacked media are presented in Section 4.

\section{Zero assignment}

In a PR, quadrature mirror (QM) filter bank, synthesis filters are completely determined by the analysis filters so that the construction of the filter bank reduces to the construction of the analysis filters [17]. The zero assignment in our method refers to the construction of FIR, QM, and minimal length analysis filters having assigned zeros at desired locations with respect to the unit circle (or at desired frequencies) [1]. We now summarize the PR, FIR, $\mathrm{QM}$, and minimal length filter bank construction method of [1].

Suppose a permitted odd filter bank delay of $n_{0}$ is given. Further suppose that $G_{0}(z)$ and $G_{1}(z)$ are two FIR transfer functions of order (number of zeros) $k$ each whose zeros coincide with the desired zeros of the analysis low-pass filter $H_{0}(z)$ and high-pass filter $H_{1}(z)$, respectively. Thus, the analysis filters will contain desired zeros if and only if

$H_{0}(z)=\hat{H}_{0}(z) G_{0}(z)$,

$H_{1}(z)=\hat{H}_{1}(z) G_{1}(z)$.

The PR condition derived in [1] is

$H_{0}(z) H_{1}(-z)-H_{0}(-z) H_{1}(z)=2 z^{-n_{0}}$.

With a further simplification in Eq. (2) the terms may be replaced with $G(z)$ and $H(z)$ where

$G(z)=G_{0}(z) G_{1}(-z)$,

$H(z)=\hat{H}_{0}(z) \hat{H}_{1}(-z)$.

Writing the terms explicitly and arranging them by Eq. (3)

$G(z) H(z)-G(-z) H(-z)=2 z^{-n_{0}}$.

For this equation to have a solution, it is necessary that the greatest common divisor of $(G(z), G(-z))$ is of the form $z^{-m}$, i.e., it should be a pure delay. In [1] it is shown that a minimal length FIR solution, $H(z)$, exits and it is unique whenever $n_{0}<4 k$ and has order at most $2 k-2$, where $k$ is the number of assigned zeros provided that $G(z)$ and $G(-z)$ are co-prime. The analysis filters are obtained by a factorization

$H(z)=\hat{H}_{0}(z) \hat{H}_{1}(-z)$,

and are in general nonunique. A hand-rule is to select the left half plane zeros in the low-pass filter and right half plane zeros in the high-pass filter [1]. The values of the $k$ assigned zeros to the low-pass filter uniquely determines the filter bank provided this hand-rule is used and it is agreed that each filter in the filter bank has order at most $2 k-2$.

Note that this is a generalization of a result of Daubechies concerning the PR, QM filter banks that correspond to Daubechies filters, in which the assigned zeros of the low-pass analysis filters are always at -1 .

As an example, suppose that the desired zeros to be assigned are at $-1,-0.8106 \pm 0.5855 i$ for the low-pass
Table 1

Filter coefficients.

\begin{tabular}{rr}
\hline$H_{1}$ & \multicolumn{1}{c}{$H_{2}$} \\
\hline 0.0581 & -0.4035 \\
-0.1140 & 0.7913 \\
-0.1423 & -0.4176 \\
0.4176 & -0.1423 \\
0.7913 & 0.1140 \\
0.4035 & 0.0581 \\
\hline
\end{tabular}

filter and at $1,0.8106 \pm 0.5855 i$ for the high-pass filter. Suppose that the duration of the allowable delay is $n_{0}=5$. Under these circumstances, a minimal order solution to Eq. (1) and its factorization according to the hand-rule described above produces the following high-pass and low-pass filters of order four each with coefficients shown in Table 1 and frequency responses given in Fig. 1.

\section{Audio and image watermarking algorithms}

In this section a new method for digital watermarking based on zero assigned filter banks is presented. The method proposed in [22] is improved by introducing wavelet decomposition into the watermarking scheme, without increasing the bandwidth requirement. Two filter banks with different assigned zeros around the stop band where each of them designates a bit ' 0 ' or ' 1 ' are used in computing the wavelet decomposition of the signal and a perceptually insignificant set of coefficients is selected by making use of the imperfections of HAS and HVS. In audio watermarking case, the fact that human ear cannot detect high frequency sounds, namely in general $20 \mathrm{kHz}$ or higher, is used and the basis to embed the watermark is chosen to be the high frequency portion of the wavelet decomposed signal. In image watermarking case, the watermark is placed around the edges as HVS is less sensitive to edges compared to flat regions. However, most compression schemes make use of the imperfections of HAS and HVS so it turns out that the most corrupted frequency band after compression is the high frequency band for audio signals and edges for image signals. Thus, watermarking becomes a complicated issue as the two most important requirements of imperceptibility and robustness against compression contradict. To overcome this problem, we merge the compression scheme in the watermarking algorithm for audio signals and use the middle frequency subbands, $L H$ or $H L$, together with a zero tree algorithm in image watermarking case.

Generally speaking our method is used in embedding a sequence of binary data in audio, gray level and color image signals. The input signal is partitioned into subblocks where a single bit is embedded into each subblock. In order to differentiate between the assigned bits, two filter banks of the same order but with different assigned zeros are constructed where each of them designates a ' 0 ' or ' 1 '. Each subblock is processed by one of the filter banks to obtain a multiresolution representation. A basis to embed the watermark is selected depending on the perceptual characteristics of the input signal and the coefficients in the basis are marked by cancellation or 

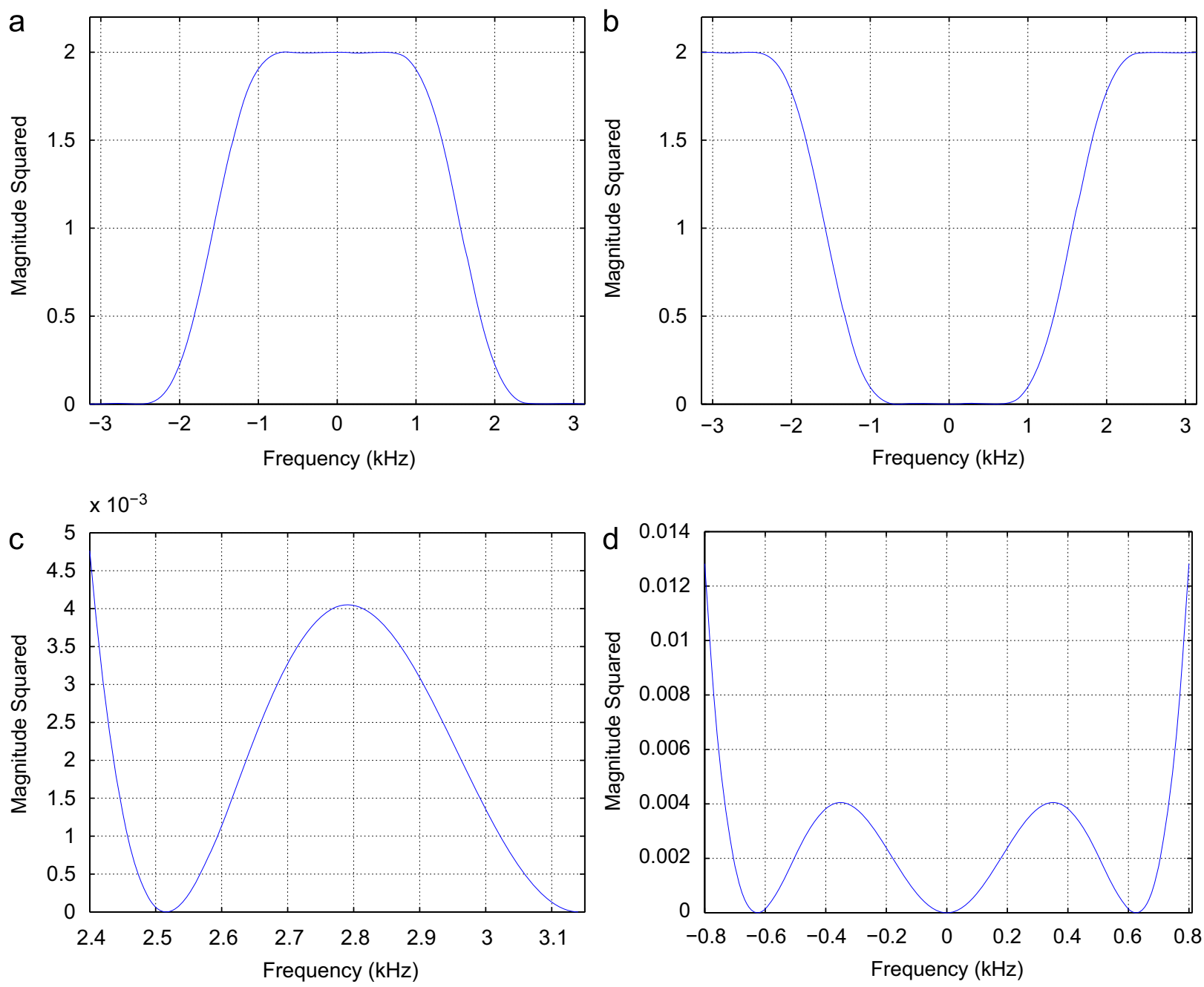

Fig. 1. Frequency responses of zero-assigned filters: (a) low-pass filter, (b) high-pass filter, (c) zoomed image around the assigned zero for LPF, (d) zoomed image around assigned zero for HPF.

replacement by some constant. In decoding, the decomposition operation is run by both of the filter banks. By looking at the coefficients in the chosen basis in the encoding phase it is determined which filter bank marked that subblock and thus which bit is embedded in it. We now go through the algorithm step by step.

The outline of this section is as follows. In the following two sections, the algorithm is explained indicating the variations in application in audio and image watermarking. The details of encoding phase is explained in Section 3.1 and the details of decoding phase are given in Section 3.2.

\subsection{Encoding}

Step I: By considering the frequency characteristics of the input signal, the two sets of assigned zeros, $z_{0}$ and $z_{1}$, where each of them has $k$ elements, are determined.

Step II: The polynomials $G_{0}(z)$ and $G_{1}(z)$ of Eq. (1) are built, where the roots of those coincide with the sets $z_{0}$ and $z_{1}$ respectively. The algorithm defined in Section 2 is applied in order to derive the two filter banks $F B_{0}$ and $F B_{1}$, where each of them designates a bit.

Step III: The input is partitioned into subblocks, or frames, of a fixed size $K$. Say these subblocks are denoted with $S_{i}$, for the image signal and $F_{i}$ for the audio signal. In color images subblocks are formed by using just the blue channel information. Here $i=1, \ldots, N$ where $N$ is the number of subblocks and corresponds to the number of bits to be embedded as a watermark.

Step IV: A stage number, $L$, is fixed to be used in the wavelet decomposition as in Fig. 3.

Step V: Each subblock $S_{i}$ or $F_{i}$ is wavelet decomposed according to the 'cascade algorithm', where either $F B_{0}$ or $F B_{1}$ is employed depending on whether ' 0 ' or ' 1 ' is the bit to be embedded [5]. An L-level multiresolution decomposition of each subblock $D_{i}, i=1, \ldots, N$ is so obtained. (In our case $L=2$ for audio watermarking and $L=3$ for image watermarking as shown in Fig. 2.)

Step VI: The best set of coefficients to embed the watermark is determined to be the perceptually insignificant coefficients in the multiresolution representation. 
In audio watermarking: The highest stage detail coefficients $D_{i}^{L}$ are chosen to be the best basis as human ear is less sensitive to high frequency sounds [16] (Fig. 3).

In image watermarking: The insignificant coefficients on $L H$ or $H L$ band of $D_{i}, i=1, \ldots, N$ are determined according to the EZW algorithm as in Fig. 4. The root location matrices $M_{i}$ are generated for each $i=1, \ldots, N$. (In our case, $L H$ band is used.)

Step VII: The watermark is embedded on the best basis by an appropriate method depending on the signal characteristics. Since the filter banks are perfectly reconstructing and the selected coefficients are not perceptually

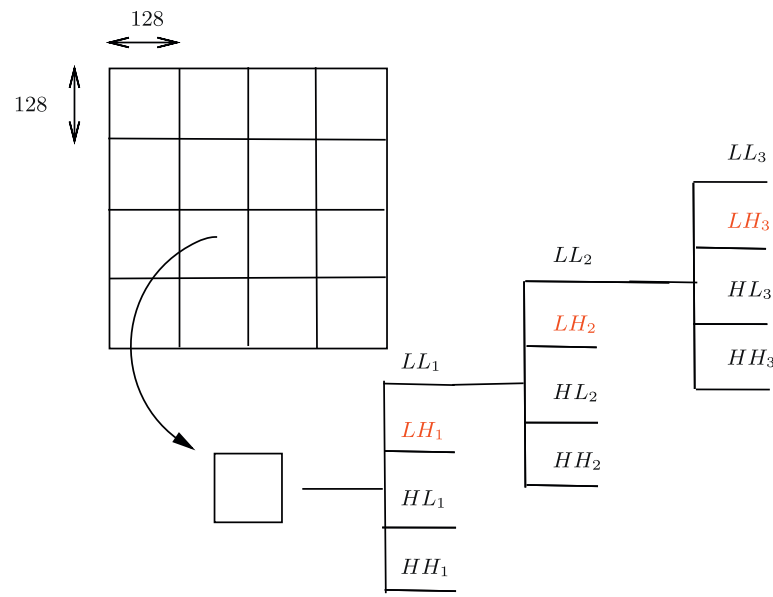

Fig. 2. Decomposition into frames and wavelet decompositions for a single frame in image watermarking.

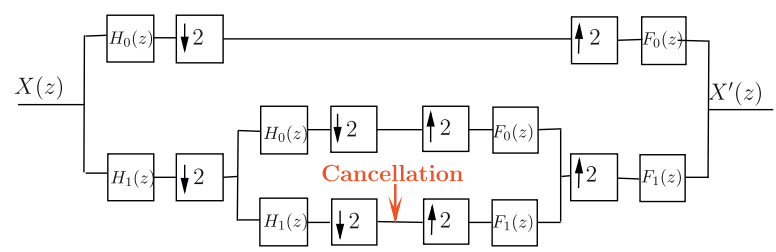

Fig. 3. $L=2$ stage implementation of the cascade algorithm.

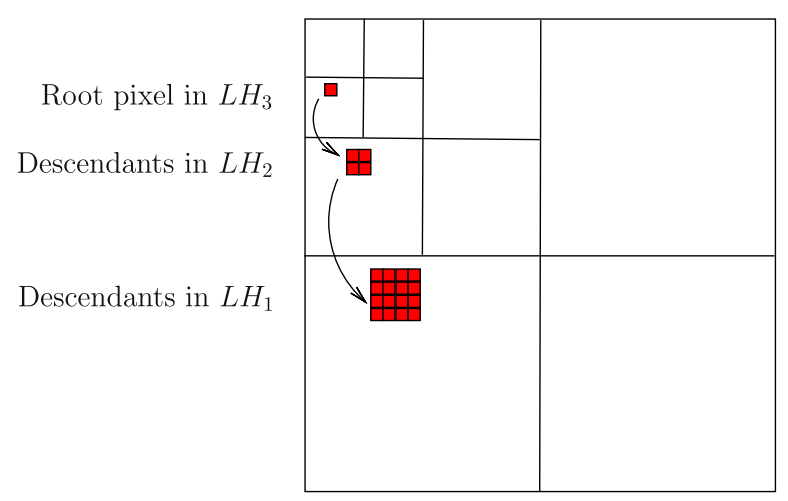

Fig. 4. Formation of a zero tree. significant, one expects that the host signal will not suffer from any significant degradation.

In audio watermarking: A compressed version of each frame is obtained by zeroing the coefficients $D_{i}^{L}$ obtained in Step VI.

In image watermarking: The zero tree elements of $D_{i}$ are replaced with some fixed number $m$ or $-m$, depending on whether $D_{i}$ is obtained using $F B_{0}$ or $F B_{1}$.

Step VIII: Simple reconstruction operation is carried out with corresponding synthesis filters according to the cascade algorithm to obtain the watermarked subblocks $\hat{F}_{i}$ or $\hat{S}_{i}$.

Step IX: The watermarked signal is the concatenation of the compressed subblocks $\hat{F}_{i}$ or $\hat{S}_{i}, i=1, \ldots, N$ of Step VIII. The sequence of ' 0 's and ' 1 's embedded in consecutive frames allows us to encode a text information in an audio file or a gray level image information in an image file.

\subsection{Decoding}

A similar procedure to embedding is employed in decoding. The required keys for decoding are the assigned zero locations, number of filter bank decomposition stages $L$, number of subblocks $N$, and size of subblocks $K$. In addition to these, in image watermarking case the root location matrices $M_{i}, i=1, \ldots, N$ are transmitted too.

Step I: The filter banks $F B_{0}$ or $F B_{1}$ are reconstructed using the assigned zero locations information and the filter bank construction algorithm of [1].

Step II: The watermarked signal is partitioned into $N$ subblocks of same size $K$ as the ones used in encoding.

Step III: Each subblock is wavelet decomposed into $D_{0 i}$ and $D_{1 i}$ using $F B_{0}$ and $F B_{1}$, respectively.

Step IV: The coefficients that are known to be at the best basis in encoding phase are checked and the ones that present a behavior closer to what is implied by the corresponding decomposition filter bank are said to be consistent so the bit that particular filter bank designates is chosen to be the extracted bit.

In audio watermarking: The bit information embedded in a frame is extracted by a comparison of the norms of highest stage detail coefficients of the two wavelet decompositions of the frame $D_{k i}$ where $i=1, \ldots, N$ and $k=0,1$. Here the norm used is given below:

$\left\|D_{k i}^{L}\right\|=\sum_{j=1}^{q}\left(d_{i j}^{L}\right)^{2}$,

where $D_{k i}^{L}$ is the $L$ th stage detail coefficients of frame $F_{i}$ obtained by filter bank $F B_{k}, k=0,1$ and $q$ denotes the size of the Lth stage detail coefficients. The ownership is verified by identifying the correct sequence of ' 0 's and ' 1 's in the consecutive frames.

In image watermarking: Using the root locations matrix $M_{i}$ both for $D_{0 i}$ and $D_{1 i}$, the mean values $m_{0 i}$ and $m_{1 i}$ of the 'previously insignificant coefficients' are computed. As illustrated in Fig. 5, if both $m_{0 i}>0$ and $m_{1 i}>0$, it may be inferred that $m_{0 i}$ implies a process by $F B_{0}$ and $m_{1 i}$ does not imply a process by $F B_{1}$ so the extracted bit is ' 1 '. In the reverse case where $m_{0 i}<0$ and $m_{1 i}<0$, simply because of the similar reason, the extracted bit is ' 0 '. On the other 


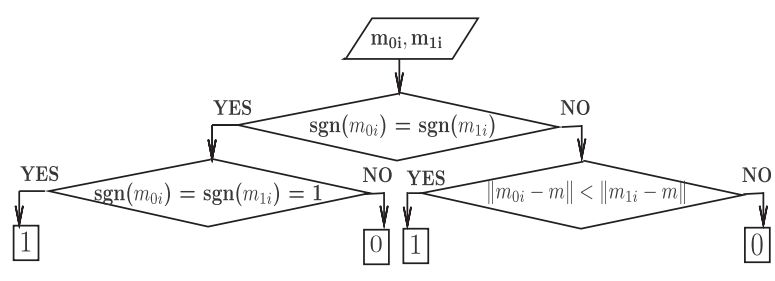

Fig. 5. Decision algorithm.

hand, when $m_{0 i}$ and $m_{1 i}$ have opposite signs and $m_{0 i}>0$ and $m_{1 i}<0$, both of these mean values imply the sign of the embedded intensity that their corresponding filter banks introduce. Hence we choose the bit that is embedded by the filter bank which has its original embedded intensity and the mean of the zero tree elements closer, i.e., when $\left|m_{0 i}-m\right|<\left|m_{1 i}+m\right|$, the extracted bit is ' 1 ', otherwise it is ' 0 '. When neither $m_{0 i}$ nor $m_{1 i}$ support the sign of the embedded intensity of its corresponding filter bank, i.e., $m_{0 i}<0$ and $m_{1 i}>0$, we again choose the filter bank which produces a result that is close to its embedded intensity. Namely, if $\left|m_{0 i}-m\right|>\left|m_{1 i}+m\right|$ the bit is determined to be ' 0 ', otherwise ' 1 '.

Obviously decoding step does not employ any correlation based scheme and thus does not require the original watermark. By this means, problems arising from a fake original or any deadlock cases are avoided.

In this section we summarized the steps of our algorithms and indicated the differences in procedure between audio and image watermarking. Next section gives the details in application and presents the results of experiments in noise free and noisy environments and under several types of attacks.

\section{Experimental results}

The algorithms defined in previous section need to be tested against several factors. First of all it must be verified that the techniques satisfy the perceptual transparency condition. It must be determined into which region the zeros can be placed without any significant artifact. Moreover, the effect of the relative positions of the zeros, i.e., the minimum distance between zeros which can be resolved must be detected. The algorithms need to also be tested against signal processing attacks, noise, compression, and estimation.

Section 4.1 presents the experimental results of the audio watermarking algorithm in noise free and noisy media and under attacks. In Section 4.1.1, the region which the zeros may be placed without any significant distortion on the host signal is determined. The robustness of the audio watermarking algorithm against the relative positions of the assigned zeros and the ability of the method to distinguish the characteristics of subblocks is investigated. Maximum tolerable SNR values are determined for various assigned zero set pairs under white Gaussian noise in Section 4.1.2. Sections 4.1.3, 4.1.4 and 4.1.5 discuss the performance of the audio watermarking method in noise-free medium, under compression and estimation type of attacks. Details about assigned zero locations, zero tree construction, stage number and frame size selections in image watermarking are presented in Section 4.2. In Sections 4.2.1, 4.2.2 and 4.2.3 the performance of the image watermarking algorithm in noise-free medium, under white Gaussian noise and under compression attack is investigated.

\subsection{Experimental results in audio watermarking}

Here some details of the experimental results for the audio watermarking algorithm described in Section 3 are presented. Mainly the following situations are considered: experiments in noise free medium, experiments under randomly generated white Gaussian noise and channel noise, and experiments under compression and estimation attacks.

We perform experiments on audio files with different sound characteristics. These files are the recordings of male and female voices and a music file together with a male voice recording with pauses. All are sampled at $22 \mathrm{kHz}$ and sample values are represented in 8 bits.

In our experiments, given the overall permitted filter bank delay $n_{0}$, a set of two filter banks are obtained by assigning $z_{0}$ or $z_{1}$ as zeros to be suppressed by the lowpass analysis filters as explained in Steps I and II of Section 3.1. The knowledge of filter bank delay $n_{0}$, assigned zero sets $z_{0}$ and $z_{1}$, stage number $L$, frame size $K$, and the watermark sequence are the keys to be provided to the validation authority for storage.

We can embed a sequence of several bits in an audio signal by dividing it into $N$ frames as in Step III of Section 3.1. Each frame is processed by one of the filter banks, $F B_{0}$ and $F B_{1}$, with different assigned zeros $z_{0}$ and $z_{1}$ depending on the bit to be assigned in that particular frame, $F_{i}, i=$ $1, \ldots, N$ as in Step IV of Section 3.1. The audio watermarks are chosen to be 4-7 letter words where each letter is represented in 7 bits in the ASCII table.

The following procedure is used to determine the zeros to be assigned. A frequency value, $f_{i}$, is determined by examining the spectra of all frames of the original audio signal. This value, $f_{i}$, should be a high frequency value at which each frame should have a nonzero component. The determination of the suppressed frequency $f_{i}$ and the magnitude of that fixes one of the zeros. In order to emphasize the suppression at that frequency, usually two or three copies of the same zero are incorporated into the low-pass filter. After choosing the frequency, $f_{i}$, and placing it on a distance $d_{i}$ from the origin, one determines the elements of to-be-assigned zero set, $z_{i}$. Once $z_{i}$ is determined, the calculation of coefficients of $G_{1}(z)$ and $G_{2}(z)$ is straightforward and the zero assignment procedure in Section 2 determines the analysis filters uniquely. Say the filter bank with a zero assigned at $z_{0}$ is $F B_{0}$ and the one with a zero assigned at $z_{1}$ is $F B_{1}$. The results presented here are obtained with the filter banks $F B_{i}, i=0,1$, each of which has a couple of assigned zeros around the stop band.

The distinction between the filters $F B_{0}$ and $F B_{1}$, and hence the distinction between the detail coefficients obtained by these different filters, is solely dependent on the choices of $z_{0}$ and $z_{1}$. Obviously, closer values for the 
elements of $z_{0}$ and $z_{1}$ will give rise to difficulties in the detection scheme. While both should be on the high frequency portion for perceptual transparency, being placed too close will cause false alarms to occur more often. In our experiments, we worked with the zeros assigned to low pass analysis filters of $F B_{i}, i=0,1$ that are separated by $1-23 \%$ of the whole spectrum, i.e. $2 \pi$, and got good results in several media. We note that, zeros may even be placed around the mid-frequency band while watermarking in noisy environments. As the noise on the watermarked signal increases, the distortion resulting from the inserted data becomes less perceptible. Thus, the add-on-noise acts like a mask for the watermark. As a result, filter banks with assigned zeros on $23 \%$ of the whole spectrum does not give rise to a perceptual degradation on noisy watermarked signals.

There is a trade-off between the number of stages of the cascade algorithm and the sound quality of the watermarked audio data. As the number of stages increases, the number of coefficients of the highest stage detail band gets smaller. Setting coefficients carrying less information to zero will yield less distortion on the watermarked signal. However, it will now be harder for the authority to detect which frequency is suppressed, and hence which bit information is embedded. The result of the comparison of the detail coefficients obtained via $F B_{0}$ and $F B_{1}$ will now be more sensitive to noise and other sources of disturbance. The number of stages to be employed in the cascade algorithm must generally be small in order for the decoding process to succeed. Nevertheless, it cannot be a single stage implementation as this will violate the perceptual transparency requirement of efficient watermarking. In order to get closer to a pure tone, the filtering process is thus carried out for two or three stages along the high frequency branch of the filter bank. After filtering the input with the high-pass decomposition filter $H_{1}(z)$ several times, components of frequency $f_{1}$ are accumulated on the lower most branch. In our experiments, we examined the effect of changing the stage number. The results for two and three stage decompositions are presented.

Although the filter banks of the cascade algorithm are determined for perfect reconstruction, the reconstructed signal would not be the same as the original one in the cascade algorithm of Fig. 3. This is because a compressed version of the original signal is fed into the synthesis part and imaging results from the upsampling in the reconstruction phase. In the decoding phase, an authority checks which frequency is suppressed in each frame by reconstructing $F B_{0}$ and $F B_{1}$ and decomposing the marked signal with both of these filter banks. In order to understand whether the highest stage detail signal is an image or not, a detection rule must be used. One possibility, followed in our application, is to compute and compare the $\mathscr{L}_{1}$ norms of the detail coefficients obtained via $F B_{0}$ and $F B_{1}$ as in Eq. (4). In our case, this method of comparison has been very successful. However, for different applications and depending on the attack or add-on noise in the transmission channel, alternate detection schemes may be employed.
A careful choice of the watermark may increase the robustness of the algorithm against false alarms during the detection process. Note that, fixing the frame size also fixes the number of bits that can be embedded in the watermarked signal, $N$. Duration of the experimented audio signal which is sampled at $22 \mathrm{kHz}$ is around $1-3 \mathrm{~s}$. In the experiments, the text to be embedded is chosen to be composed of a word with several letters, generally 4-7 letters. For a 6-letter word, 42 bits must be embedded as each letter is represented in 7 bits in the ASCII table. For each bit a frame of 128,256 or 512 samples is taken. This allows us, e.g., to embed about 12 copies of a 6-letter word in a 3-s signal if we use frames of size 128. This would tolerate one or two false diagnosis of the text in, say, noisy environments.

We now go into the details of implementation and present the results of our experiments. In Section 4.1.1 the effect of placement of the assigned zeros is investigated from the aspect of perceptual transparency and the ability to distinguish between closely placed zeros. In Section 4.1.2, robustness against noise is investigated first by modeling the noise to be a white Gaussian one and then by using a recording on a voiceless wireless telephone channel. Robustness against compression attack and estimation type of attacks is discussed in Sections 4.1.4 and 4.1.5.

\subsubsection{Experiments in noise free medium}

In this section effects of stage number selection, frame size selection and placement of zeros are examined in noise free environment.

The low pass analysis filters of $F B_{0}$ and $F B_{1}$ have one zero fixed at -1 . The other assigned zero of the low pass analysis filter of $F B_{0}$ is fixed at $10 \%$ vicinity of $2 \pi$, i.e., at $\{1 \angle(1 \pm 0.1) 2 \pi\}$ with multiplicity of 2 . The assigned zero of the low pass analysis filter of $F B_{1}$ is located at $3 \%, 5 \%, 7 \%$ or $9 \%$ vicinity of $2 \pi$ with multiplicity 2 . The effect of these alternative zero configurations on bit reliability (BR) is examined.

In Table 2 the first column denotes the number of decomposition stages $L$ and the second column denotes the frame size $K$. At the third column the argument of the assigned zero of the low pass filter (LPF) of $F B_{1}, f_{1}$, is given in terms of percentages of $2 \pi$. For instance in row 1 of Table 2, argument of the assigned zero is noted as 3 , which means that the LPF has assigned zeros at $\{-1,1 \angle(1 \pm 0.03) 2 \pi\}$, where the term $\{1 \angle(1 \pm 0.03) 2 \pi\}$ has multiplicity two.

Our method works with $100 \%$ bit reliability even when the zeros are placed $2 \%$ apart on the unit circle. Only when two zeros are on $\{1 \angle(1 \pm 0.09) 2 \pi\}$ and the other two are on $\{1 \angle(1 \pm 0.1) 2 \pi\}$, the bit reliability decreases to 0.964 for the experiment on male voice recording with three stage decomposition scheme. Note that as our watermarking algorithm allows us to insert multiple copies of the text into the audio signal, missing out a few bits in the detection stage still provides many correct copies of text to be detected. On the other hand, it should be noted that as long as the perceptual transparency condition is satisfied, there is no absolute necessity to place zeros too close to each other (Table 3 ). 
Table 2

Bit reliabilities in extraction of watermark in noise free medium.

\begin{tabular}{|c|c|c|c|c|c|c|}
\hline \multicolumn{3}{|c|}{ Encoding parameters } & \multicolumn{4}{|l|}{$\mathrm{BR}$} \\
\hline$L$ & $K$ & $f_{1}$ & Male voice & Female voice & Music & Male voice with pauses \\
\hline 2 & 128 & 3 & 1 & 1 & 1 & 1 \\
\hline 2 & 128 & 4 & 1 & 1 & 1 & 1 \\
\hline 2 & 128 & 5 & 1 & 1 & 1 & 1 \\
\hline 2 & 128 & 6 & 1 & 1 & 1 & 1 \\
\hline 2 & 128 & 7 & 1 & 1 & 1 & 1 \\
\hline 2 & 128 & 8 & 1 & 1 & 1 & 1 \\
\hline 2 & 128 & 9 & 1 & 1 & 1 & 1 \\
\hline 2 & 256 & 3 & 1 & 1 & 1 & 1 \\
\hline 2 & 256 & 4 & 1 & 1 & 1 & 1 \\
\hline 2 & 256 & 5 & 1 & 1 & 1 & 1 \\
\hline 2 & 256 & 6 & 1 & 1 & 1 & 1 \\
\hline 2 & 256 & 7 & 1 & 1 & 1 & 1 \\
\hline 2 & 256 & 8 & 1 & 1 & 1 & 1 \\
\hline 2 & 256 & 9 & 1 & 1 & 1 & 1 \\
\hline 2 & 128 & 3 & 1 & 1 & 1 & 1 \\
\hline 3 & 128 & 4 & 1 & 1 & 1 & 1 \\
\hline 3 & 128 & 5 & 1 & 1 & 1 & 1 \\
\hline 3 & 128 & 6 & 1 & 1 & 1 & 1 \\
\hline 3 & 128 & 7 & 1 & 1 & 1 & 1 \\
\hline 3 & 128 & 8 & 1 & 0.96 & 1 & 1 \\
\hline 3 & 128 & 9 & 0.96 & 0.96 & 1 & 1 \\
\hline 3 & 256 & 3 & 1 & 1 & 1 & 1 \\
\hline 3 & 256 & 4 & 1 & 1 & 1 & 1 \\
\hline 3 & 256 & 5 & 1 & 1 & 1 & 1 \\
\hline 3 & 256 & 6 & 1 & 1 & 1 & 1 \\
\hline 3 & 256 & 7 & 1 & 1 & 1 & 1 \\
\hline 3 & 256 & 8 & 1 & 1 & 1 & 1 \\
\hline 3 & 256 & 9 & 1 & 1 & 1 & 1 \\
\hline
\end{tabular}

Table 3

Bit reliabilities for male and female voices and music signal under channel noise decomposed into two stages with frame size 128 .

\begin{tabular}{|c|c|c|c|c|c|}
\hline \multicolumn{3}{|c|}{ Encoding parameters } & \multicolumn{3}{|l|}{ BR } \\
\hline$L$ & $K$ & $f_{1}$ & Male voice & Female voice & Music \\
\hline 2 & 128 & 3 & 1 & 1 & 1 \\
\hline 2 & 128 & 5 & 1 & 1 & 1 \\
\hline 2 & 128 & 7 & 1 & 1 & 1 \\
\hline 2 & 128 & 9 & 1 & 1 & 1 \\
\hline 2 & 256 & 3 & 1 & 1 & 1 \\
\hline 2 & 256 & 5 & 1 & 1 & 1 \\
\hline 2 & 256 & 7 & 1 & 1 & 1 \\
\hline 2 & 256 & 9 & 1 & 1 & 1 \\
\hline 3 & 128 & 3 & 1 & 1 & 1 \\
\hline 3 & 128 & 5 & 1 & 1 & 1 \\
\hline 3 & 128 & 7 & 1 & 0.96 & 0.96 \\
\hline 3 & 128 & 9 & 0.78 & 0.71 & 0.71 \\
\hline 3 & 256 & 3 & 1 & 1 & 1 \\
\hline 3 & 256 & 5 & 1 & 1 & 1 \\
\hline 3 & 256 & 7 & 1 & 1 & 1 \\
\hline 3 & 256 & 9 & 1 & 0.96 & 0.96 \\
\hline
\end{tabular}

\subsubsection{Experiments under randomly generated Gaussian noise}

Gaussian noise with a mean of 0 and a variance of 1 , is added on top of the watermarked signal and the success rate in extracting the watermark from the attacked signal is investigated.

As in case of the experiments in noise free medium, filter banks with assigned zeros of multiplicity two are used in the two and three stage decomposition schemes while the frame size $K$ is either 128 or 256 . The arguments of the assigned zeros of $F B_{0}$ and $F B_{1}$ range between $1 \%$ and $23 \%$ of $2 \pi$, i.e., they are in the interval $\{1 \angle(1 \pm 0.01) 2 \pi\}-\{1 \angle(1 \pm 0.23) 2 \pi\}$.

Typical SNR values for testing the robustness of an audio watermark are chosen to be as the ones in [9]. As expected, bit reliability increases with decreasing SNR. It reaches up to $80 \%$ when SNR is equal to $50 \mathrm{~dB}$ while it is around $45 \%$ when SNR is equal to $20 \mathrm{~dB}$. Choosing the frame size $K$ to be 128 and stage number $L$ to be 2 produces better results.

We define the term tolerable SNR to be the SNR value for which the method extracts the watermark with at least 95\% bit reliability. From Fig. 6 it is clear that when the two assigned zeros of filter banks $F B_{0}$ and $F B_{1}$ are close, i.e., around $x=y$ line, the tolerable SNR is higher than other portions where the distance between assigned zeros are larger. Moreover, one should note that on $x=y$ line, tolerable SNR is higher when the two assigned zeros have small arguments. As one follows the line to the higher values of assigned zero arguments, the tolerable SNR decreases. Namely, it is seen in Fig. 6 that, as the frame size increases, the detection procedure gets more sensitive to the add-on noise when the zeros are located close 


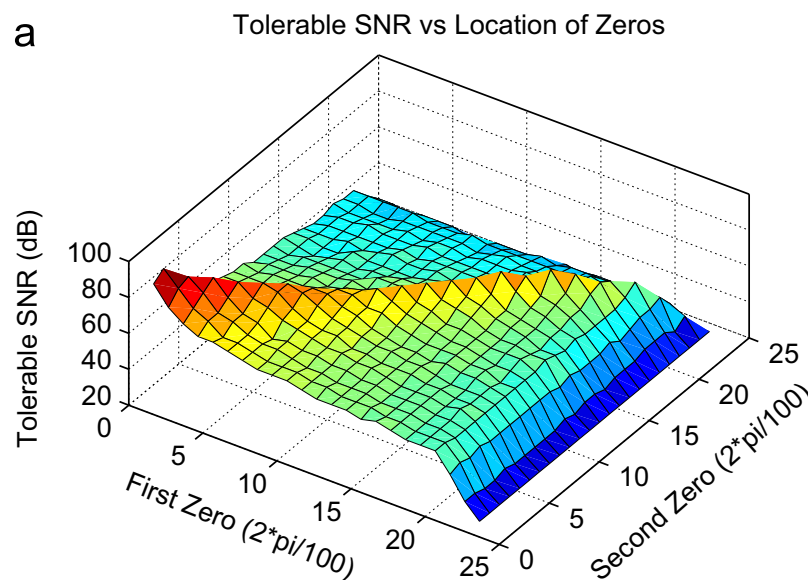

C

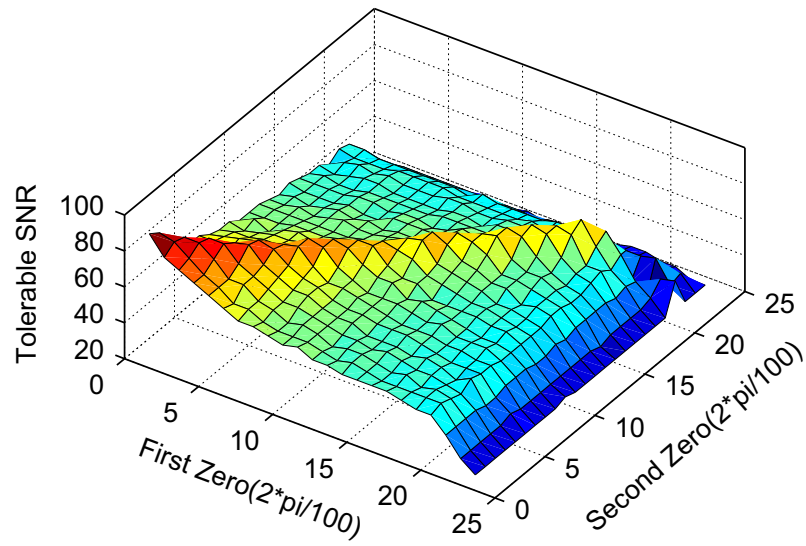

b

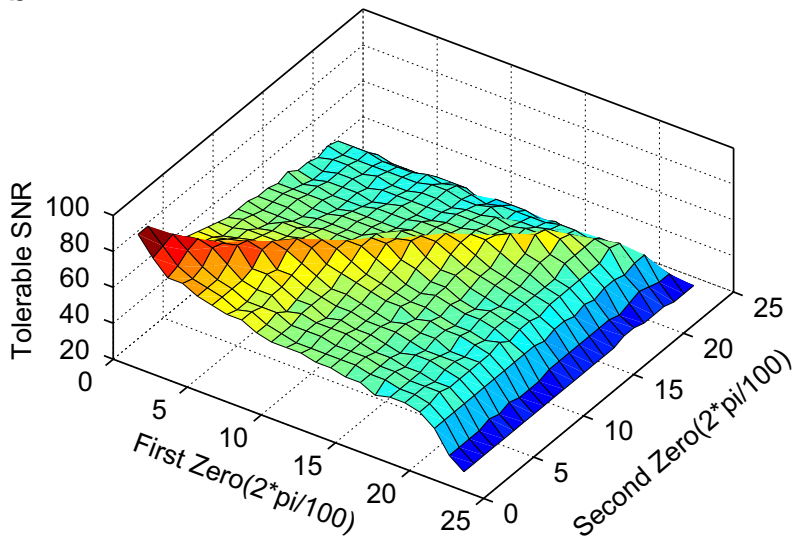

d

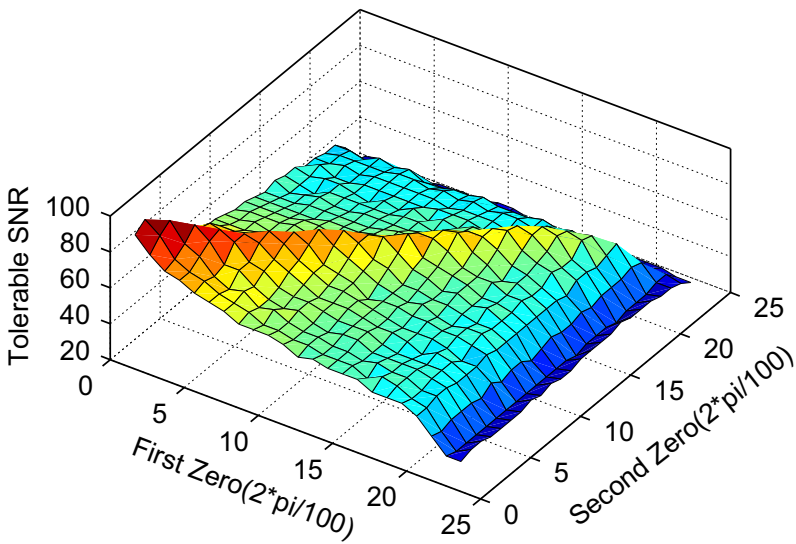

Fig. 6. Tolerable SNR for male voice partitioned into frames of (a) $128 \times 128$ and (b) $256 \times 256$ and for female voice partitioned into frames of (c) $128 \times 128$ and (d) $256 \times 256$ processed with 2 stage filter banks.

around the lower frequency values. On the other hand, the zeros that are close around the higher frequencies lead to a detection scheme, which is less sensitive to channel noise. Moreover, when zeros are placed most apart, the bit reliability gets higher for smaller frames.

\subsubsection{Experiments under channel noise}

In this section, the success rate in extracting the watermark from a marked signal with an add-on channel noise that is chosen to be a recording on a voiceless wireless telephone channel is examined. It is observed that only when arguments of the assigned zeros are too close, i.e., $1-3 \%$ of $2 \pi$ apart on the unit circle bit reliability decreases to $71-96 \%$, otherwise it is $100 \%$ for any stage number or frame size selection.

\subsubsection{Experiments under compression attack}

Watermarked signals are converted to MP3 format at $144 \mathrm{Kbps}$ and regular detection scheme is employed. It is observed that compression causes a fair decrease in bit reliability. Bit reliability changes between 0.4 and 0.5 for all cases. It is obvious that the method is quite fragile against MPEG compression attack and needs to be strengthened. One approach may be to employ EZW in selecting the set of coefficients to be marked. However, the zero tree structure of the image watermarking algorithm does not work well. The architecture of the zero tree may be modified as in the case of [6].

\subsubsection{Estimation type of attacks}

Estimation type of attacks assumes that the watermark can be estimated without prior knowledge of the embedding rule or embedding keys. The watermark is considered to be noise and a denoising scheme is employed [18]. Low pass filtering seems a common approach for denoising. Our proposed method is fragile under low pass filtering attack and hence needs to be strengthened. One counter measure against low pass filtering is to employ a notch filter on a low frequency component in wavelet decomposed signal. A notch filter of narrow stop band can be shown not to degrade the perceptual quality of the signal. Moreover, the watermark embedded by the notch filter can be detected efficiently.

In Table 4 the details about the performance of notch filter support are presented. The first column denotes the frame size $K$. In our experiments, the assigned zero of $F B_{0}$ is kept fixed at $1 \%$ vicinity of $2 \pi$, i.e., at $\{1 \angle(1 \pm 0.01) 2 \pi\}$. The second column denotes the argument of the assigned 
Table 4

Bit reliability for male voice decomposed in 1 and 2 stages with notch filter support under low pass filtering attack.

\begin{tabular}{|c|c|c|c|}
\hline \multicolumn{2}{|c|}{ Encoding parameters } & \multirow{2}{*}{$\begin{array}{l}1 \text { stage performance } \\
\text { BR }\end{array}$} & \multirow{2}{*}{$\begin{array}{l}2 \text { stages performance } \\
\text { BR }\end{array}$} \\
\hline$K$ & $f_{1}$ & & \\
\hline 128 & 2 & 0.89 & 0.89 \\
\hline 128 & 3 & 0.89 & 0.89 \\
\hline 128 & 4 & 0.89 & 0.89 \\
\hline 128 & 5 & 0.89 & 0.89 \\
\hline 128 & 6 & 0.89 & 0.89 \\
\hline 128 & 7 & 0.92 & 0.92 \\
\hline 128 & 8 & 0.92 & 0.92 \\
\hline 128 & 9 & 0.92 & 0.92 \\
\hline 128 & 10 & 0.92 & 0.92 \\
\hline 128 & 11 & 0.92 & 0.92 \\
\hline 128 & 12 & 0.92 & 0.92 \\
\hline 128 & 13 & 0.92 & 0.92 \\
\hline 128 & 14 & 0.92 & 0.92 \\
\hline 256 & 2 & 0.78 & 0.78 \\
\hline 256 & 3 & 0.78 & 0.78 \\
\hline 256 & 4 & 0.78 & 0.78 \\
\hline 256 & 5 & 0.78 & 0.78 \\
\hline 256 & 6 & 0.78 & 0.78 \\
\hline 256 & 7 & 0.78 & 0.78 \\
\hline 256 & 8 & 0.78 & 0.78 \\
\hline 256 & 9 & 0.78 & 0.78 \\
\hline 256 & 10 & 0.78 & 0.78 \\
\hline 256 & 11 & 0.78 & 0.78 \\
\hline 256 & 12 & 0.78 & 0.78 \\
\hline 256 & 13 & 0.78 & 0.78 \\
\hline 256 & 14 & 0.78 & 0.78 \\
\hline
\end{tabular}

zero of $F B_{1}$. For instance in the first line 2 implies that the assigned zero of $F B_{1}$ is at $\{1 \angle(1 \pm 0.02) 2 \pi\}$. In column three, there is the bit reliability of decoding after extracting the watermark from a marked signal which is attacked by a 5th order Butterworth low pass filter with a cut-off frequency of $0.95 \pi$.

Our watermark extraction experiment with different types of speech signals indicate about $90 \%$ bit reliability provided the stop band of the notch filter is determined taking the frequency characteristics of the set of signals to be watermarked into account.

\subsection{Experimental results in image watermarking}

In this section we give the details in the application of our image watermarking algorithm such as zero locations and the properties of the input image. The success rate in noise free environments is presented in Section 4.2.1. The algorithm is tested under randomly generated white Gaussian noise with a mean of 0 and variance of 1 and the results are given in Section 4.2.2, whereas performance of the method under compression attack is investigated in Section 4.2.3.

To satisfy the perceptual transparency requirement and provide robustness against attacks, the zero trees are constructed on $L H$ or $H L$ frequency bands [14] and the tree elements are replaced as in Step VII of Section 3.1, depending on the assigned bit with the embedded intensity being either $m$ or $-m$, where $m$ is chosen to be the $5 \%$ of the maximum coefficient on the highest branch of the chosen frequency band [24]. Conceptually, the

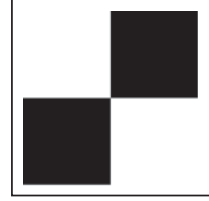

Fig. 7. An example image watermark.

embedded watermark in an image is as shown in Fig. 7, where the diagonal frames correspond to bits ' 0 ' and the off-diagonal frames to bits ' 1 '.

From the aspect of storage requirements and security, our method brings many advantages. The argument, $f_{i}$, and the magnitude, $d_{i}$, of the assigned zero is enough to compute the filter coefficients so this not only decreases the amount of transmitted data, i.e the bandwidth, but also makes the algorithm more secure unless the design procedure is available to anyone. Nevertheless one should note that the locations of the insignificant coefficients must be stored in a simple binary matrix against any attack on the watermarked image. Attacks may change the roots, hence resulting in a different tree. Nevertheless the computational complexity or storage space does not cost much, since it is enough to keep the root locations as the whole tree can be reconstructed from the roots and since the size of the location matrix is $\left(\frac{1}{8}\right)$ th of the original image as downsampling operations in the filter bank structure half the size at every stage.

In our experiments, we used the conventional image of 'Lena' because it contains details, flat regions, shading, and texture. After a few experiments we found out that for this method the most efficient scheme on 'Lena' is observed when the number of decomposition levels $L=3$ and partitions $S_{i}, i=1, \ldots, N$ are of size $128 \times 128$. Furthermore the threshold for detecting the insignificant coefficients is chosen to be $5 \%$ of the maximum coefficient in absolute value in the 3 rd detail band, i.e., $D_{0 i}^{3}$ or $D_{1 i}^{3}$.

\subsubsection{Performance in noise free medium}

In Fig. 8 the original and watermarked images of $512 \times$ 512 'Lena' , 'Barbara' , and 'Peppers' are presented. In Fig. 7 the corresponding watermark can be seen. In obtaining the watermarked image in Fig. 8, filter banks $F B_{0}$ and $F B_{1}$ consisting of filters of order 5 each are used. For this particular case, a filter bank with a low pass decomposition filter which has an assigned zero on $\{1 \angle(1 \pm 0.01) 2 \pi\}$ is used in the watermarking process together with a filter bank with a low pass decomposition filter which has an assigned zero on $\{0.7 \angle(1 \pm 0.13) 2 \pi\}$.

Throughout the experiments the angles of the assigned zeros run from $\pi \pm 2 \pi / 100$ to $\pi \pm 26 \pi / 100$. The distance of the assigned zeros to the origin varies between 1 and 0.7 . It is observed that for these assigned zero locations there is no perceptual degradation on the watermarked image.

In Table 5 the PSNR of the marked image for corresponding zero configurations is presented. For instance, in column one of Table 5 , the value 2 corresponds to $\{1 \angle(1 \pm 0.02) 2 \pi\}$ and is the value of the argument of the assigned zero of $F B_{0}$. Similarly, column 
a

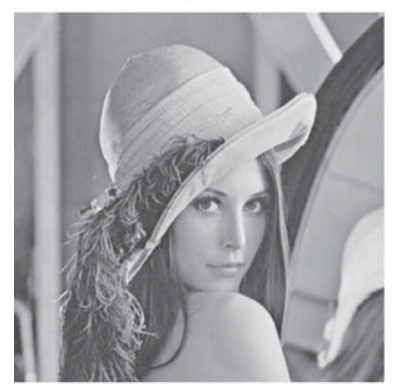

C

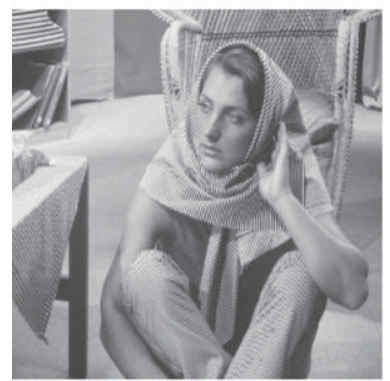

e

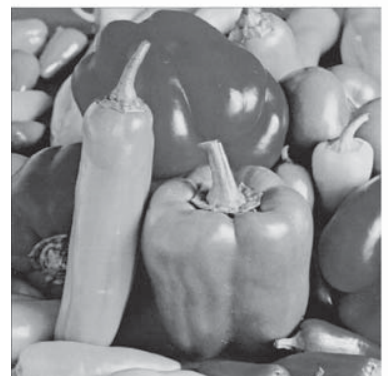

Fig. 8. (a), (c), (e) Original images of Lena, Barbara and Peppers. (b), (d), (f) Corresponding watermarked images.

two denotes the magnitude of the assigned zero. The last three columns show the peak signal-to-noise ratio when the image is watermarked by those two filter banks. It is observed that PSNR values are high enough so the watermark may be claimed to be transparent.

After indicating the details about encoding phase explained in Section 3.1 and pointing out the practical advantages of our image watermarking method, we now present the performance of the algorithm against white Gaussian noise and JPEG compression in Sections 4.2.2 and 4.2.3.

\subsubsection{Robustness against white Gaussian noise}

This section presents the success rates in extracting the watermark from a signal that is attacked by additive white Gaussian noise.

The exact watermark can be extracted even under exposure to a high white Gaussian noise with zero mean and unit variance. In Table 6 for the indicated noise values and corresponding assigned zero frequencies, where all
Table 5

PSNR on the watermarked images.

\begin{tabular}{llllll}
\hline \multicolumn{2}{l}{ Encoding parameters } & & \multicolumn{3}{l}{ PSNR } \\
\cline { 1 - 3 }$f_{0}$ & $d_{0}$ & & Lena & Barbara & Peppers \\
\hline 2 & 1 & & 69.09 & 61.45 & 51.20 \\
3 & 1 & & 69.06 & 59.44 & 49.17 \\
4 & 1 & & 67.49 & 58.63 & 47.99 \\
5 & 1 & & 68.40 & 63.80 & 55.96 \\
1 & 0.9 & & 69.32 & 61.33 & 51.03 \\
2 & 0.9 & & 69.18 & 59.40 & 49.12 \\
3 & 0.9 & & 67.21 & 58.57 & 47.89 \\
4 & 0.9 & & 68.71 & 63.65 & 55.43 \\
5 & 0.9 & & 69.40 & 61.20 & 50.73 \\
1 & 0.8 & & 69.33 & 59.52 & 49.02 \\
2 & 0.8 & & 67.17 & 58.49 & 48.16 \\
3 & 0.8 & & 69.19 & 63.37 & 54.79 \\
4 & 0.8 & & 69.85 & 60.94 & 49.97 \\
5 & 0.8 & 69.14 & 59.30 & 48.86 \\
1 & 0.7 & 67.06 & 58.28 & 48.09 \\
2 & 0.7 & 69.74 & 62.70 & 53.96 \\
3 & 0.7 & 70.33 & 60.40 & 49.63 \\
4 & 0.7 & 69.18 & 59.00 & 48.78 \\
5 & 0.7 & 66.83 & 58.05 & 47.99 \\
\hline
\end{tabular}

Table 6

SNR of white Gaussian noise.

\begin{tabular}{|c|c|c|c|c|c|c|c|}
\hline \multicolumn{2}{|c|}{ Encoding parameters } & \multicolumn{6}{|c|}{ SNR of Gaussian noise } \\
\hline \multirow[t]{2}{*}{$f_{0}$} & \multirow[t]{2}{*}{$f_{1}$} & \multicolumn{2}{|l|}{ Lena } & \multicolumn{2}{|c|}{ Barbara } & \multicolumn{2}{|c|}{ Peppers } \\
\hline & & $d_{0}=1$ & $d_{0}=0.8$ & $d_{0}=1$ & $d_{0}=0.8$ & $d_{0}=1$ & $d_{0}=0.8$ \\
\hline 10 & 10 & * & -5.4 & * & -7.6 & * & -6.2 \\
\hline 10 & 20 & -4.6 & -7.0 & -5.8 & -4.0 & -7.8 & -3.8 \\
\hline 10 & 30 & -8.2 & -4.2 & -5.6 & -6.2 & -6.6 & -7.2 \\
\hline 10 & 40 & -7.0 & -6.0 & -5.0 & -3.2 & -4.0 & -7.6 \\
\hline 10 & 50 & -6.4 & -4.6 & -2.6 & -5.6 & -4.4 & -8.8 \\
\hline 20 & 10 & -5.6 & -4.2 & -6.6 & -8.0 & -7.6 & -7.0 \\
\hline 20 & 20 & * & -4.4 & $*$ & -4.8 & $*$ & -8.8 \\
\hline 20 & 30 & -6.0 & -9.0 & -4.8 & -6.2 & -8.8 & -7.0 \\
\hline 20 & 40 & -6.0 & -5.6 & -6.4 & -5.8 & -6.8 & -6.6 \\
\hline 20 & 50 & -5.0 & -8.2 & -8.2 & -6.2 & -5.6 & -9.8 \\
\hline 30 & 10 & -5.2 & -7.6 & -3.6 & -6.0 & -5.6 & -8.6 \\
\hline 30 & 20 & -7.6 & -7.6 & -6.8 & -3.6 & -5.2 & -6.6 \\
\hline 30 & 30 & * & -5.6 & $*$ & -4.6 & * & -7.0 \\
\hline 30 & 40 & -6.4 & -5.8 & -6.2 & -3.8 & -5.2 & -8.2 \\
\hline 30 & 50 & -8.2 & -4.6 & -7.4 & -6.6 & -2.8 & -7.0 \\
\hline 40 & 10 & -6.0 & -8.6 & -5.8 & -3.0 & -3.6 & -7.6 \\
\hline 40 & 20 & -3.8 & -5.4 & -3.0 & -6.2 & -5.8 & -5.4 \\
\hline 40 & 30 & -4.6 & -6.4 & -7.0 & -6.0 & -6.6 & -6.6 \\
\hline 40 & 40 & * & -9.4 & * & -4.0 & * & -9.4 \\
\hline 40 & 50 & -5.8 & -7.0 & -5.8 & -4.4 & -5.8 & -6.4 \\
\hline 50 & 10 & -2.4 & -8.2 & -5.0 & -1.8 & -4.8 & -5.2 \\
\hline 50 & 20 & -2.0 & -7.2 & -4.8 & -6.4 & -7.0 & -7.8 \\
\hline 50 & 30 & -7.0 & -7.4 & -3.8 & -5.2 & -6.4 & -5.2 \\
\hline 50 & 40 & -7.0 & -4.6 & -10.2 & -2.6 & -6.8 & -5.6 \\
\hline 50 & 50 & * & -5.6 & $*$ & -2.8 & * & -6.2 \\
\hline
\end{tabular}

the magnitudes are equal to 1 , the method extracts the watermark with $100 \%$ success, i.e. BER is 0 for all cases. In Table 6 , the mean signal-to-noise ratio (SNR) which the method achieves full success is -6.0978 , -5.3111 , and -6.5067 for images Lena, Barbara and Peppers respectively (Fig. 9). 
a

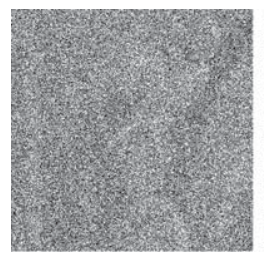

b

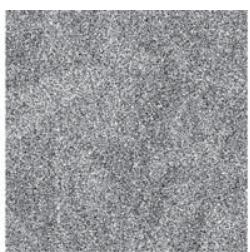

C

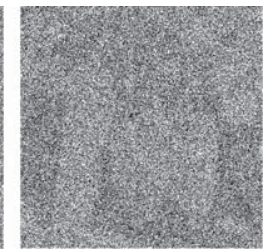

Fig. 9. Watermarked images of Lena, Barbara, and Peppers with noise on top.

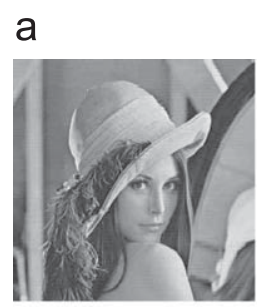

b

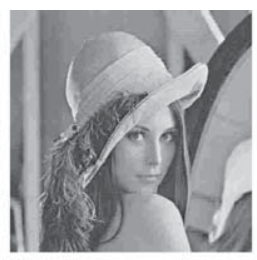

d

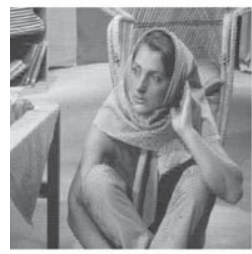

$\mathrm{g}$

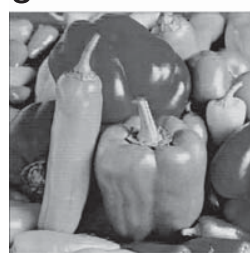

e

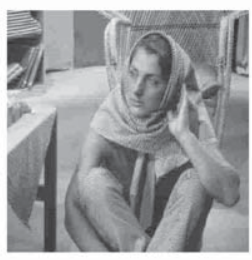

h
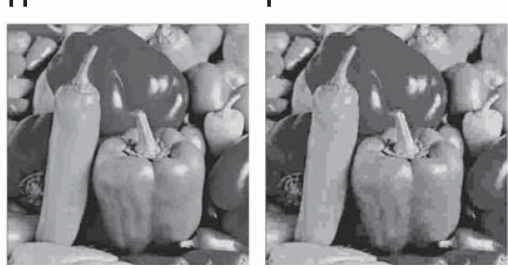

Fig. 10. Compressed images of above watermarked images with qualities $20 \%$ ((a), (d), (g)), 10\% ((b), (e), (h)) and 5\% ((c), (f), (i)).

In [23], the experiments on audio watermarking show that as the zeros of the filter banks get apart from each other it becomes easier to determine which filter bank marks the frame. However, in this case there is no regular behavior about the angles. This phenomenon may be explained with the fact that as in audio watermarking case the detail coefficients are set to zero, the distinction of the frequency response of the filter banks exhibits a more crucial effect. However, in our image watermarking method, embedded intensity replaces the zero tree coefficients thus assigned zero locations do not play such a significant role in identification.

\subsubsection{Robustness against compression}

The first international compression standard for continuous tone still images is the JPEG compression standard [19]. Here we compress the marked signal in certain JPEG quality levels and the success rates for several assigned zero locations are presented.
Table 7

PSNR and bit error rate in JPEG compressed Lena and Barbara.

\begin{tabular}{|c|c|c|c|c|c|c|c|c|}
\hline \multirow[t]{3}{*}{$Q$} & \multicolumn{4}{|l|}{ Lena } & \multicolumn{4}{|c|}{ Barbara } \\
\hline & \multicolumn{2}{|c|}{$f_{0}=20$} & \multicolumn{2}{|c|}{$f_{0}=30$} & \multicolumn{2}{|c|}{$f_{0}=20$} & \multicolumn{2}{|c|}{$f_{0}=30$} \\
\hline & PSNR & BER & PSNR & BER & PSNR & BER & PSNR & BER \\
\hline 90 & 51.92 & 0 & 52.68 & 0 & 42.27 & 0 & 42.21 & 0 \\
\hline 80 & 50.34 & 0 & 50.39 & 0 & 37.30 & 0 & 37.18 & 0 \\
\hline 70 & 38.16 & 0 & 38.13 & 0 & 34.62 & 0 & 34.64 & 0 \\
\hline 60 & 34.61 & 0 & 34.56 & 0 & 32.30 & 0 & 32.23 & 0 \\
\hline 50 & 32.90 & 0 & 32.92 & 0 & 30.79 & 0 & 30.78 & 0 \\
\hline 40 & 32.43 & 0 & 32.37 & 0 & 29.69 & 0 & 29.71 & 0 \\
\hline 30 & 30.14 & 0 & 30.14 & 0 & 27.66 & 0 & 27.66 & 0 \\
\hline 20 & 27.61 & 0 & 27.67 & 0 & 25.11 & 0 & 25.13 & 0 \\
\hline 10 & 22.91 & 0.25 & 22.96 & 0 & 21.60 & 0 & 21.61 & 0 \\
\hline 5 & 17.84 & 0.50 & 17.87 & 0.50 & 17.74 & 0.75 & 17.73 & 0.75 \\
\hline 2 & 12.53 & 0.50 & 12.47 & 0.50 & 13.24 & 0.75 & 13.24 & 0.75 \\
\hline 1 & 12.53 & 0.50 & 12.47 & 0.50 & 13.30 & 0.75 & 13.28 & 0.75 \\
\hline
\end{tabular}

Table 8

PSNR and bit error rate in JPEG compressed Peppers.

\begin{tabular}{|c|c|c|c|c|}
\hline \multirow[t]{2}{*}{$Q$} & \multicolumn{2}{|l|}{$f_{0}=2$} & \multicolumn{2}{|c|}{$f_{0}=30$} \\
\hline & PSNR & BER & PSNR & BER \\
\hline 90 & 38.2381 & 0 & 38.22 & 0 \\
\hline 80 & 36.6924 & 0 & 36.73 & 0 \\
\hline 70 & 34.5183 & 0 & 34.54 & 0 \\
\hline 60 & 31.6490 & 0 & 31.74 & 0 \\
\hline 50 & 29.6901 & 0 & 29.24 & 0 \\
\hline 40 & 27.8821 & 0 & 27.88 & 0 \\
\hline 30 & 28.1564 & 0 & 28.16 & 0 \\
\hline 20 & 25.2628 & 0 & 25.29 & 0 \\
\hline 10 & 19.1654 & 0.25 & 19.07 & 0.25 \\
\hline 5 & 15.3548 & 0.5 & 15.36 & 0.5 \\
\hline 2 & 12.1842 & 0.5 & 12.18 & 0.5 \\
\hline 1 & 12.1629 & 0.5 & 12.16 & 0.5 \\
\hline
\end{tabular}

In Fig. 10 three compressed images which are the JPEG compressed versions of the watermarked image in Fig. 8 are presented. Though the images are highly corrupted the watermark is still extracted with $100 \%$ success.

In Tables 7 and 8, the first column indicates the JPEG compression quality in percentages. The PSNR and BER are presented for the denoted encoding parameters.

Watermarked images are tested against JPEG compression at qualities from $90 \%$ down to $10 \%$. In Tables 7 and 8 , it is observed that down to $10 \%$ compression quality, the watermark is extracted perfectly but at $10 \%$ or $5 \%$ compression quality, one partition may be detected with a wrong bit. For some zero configurations successful results are obtained for $10 \%$ compression quality.

\section{Conclusion}

We have presented the image and audio watermarking algorithms step by step by indicating the differences in implementation in Section 3 and pointed out the practical details about selections of several parameters such as frame size, decomposition stage number, $L$, and the 
threshold for zero tree condition in Sections 4.1 and 4.2. The rest of this section is dedicated to the experimental results in noise free and noisy environments and under attacks.

In noise free environment, the region on the complex plane where any assigned zero that does not lead to a significant artifact on the marked image may be placed is determined. Moreover, the experiments in noise free environment test the robustness of the audio and image watermarking techniques against relative positions of elements of the assigned zero sets, $z_{0}$ and $z_{1}$, of filter banks $F B_{0}$ and $F B_{1}$. The results indicate that just when the elements of the two assigned zero sets are too close, misdetections may take place. However, note that for other selections $100 \%$ success is achieved in detection.

In noisy environment, the robustness of the method against white Gaussian noise and channel noise is investigated. For image watermarking, tolerable SNR which is the noise level that does not lead to more than 5\% misdetection rate when imposed onto a marked signal, is defined for several assigned zero set couples. It is observed that even when the noisy marked signal is not intelligible, the watermark may be extracted with more than $95 \%$ bit reliability. Under channel noise the audio watermarking algorithm performs with $100 \%$ success except for assigned zeros which are too close on complex plane.

The results of the experiments under compression attack indicate that the audio watermarking algorithm is fragile against MPEG. The image watermarking algorithm produces better results than several previously defined techniques and can yield $100 \%$ success rate even when compressed with $10 \%$ compression quality.

Image watermarking via zero assigned filter banks has advantages over the previously proposed techniques in several respects. Under white Gaussian noise and compression attacks, the proposed method improves both PSNR properties in comparison to $[11,12,2]$. The degradations caused by encoding is much less and the method is more robust against compression and when exposed to high white Gaussian noise.

Compared to the techniques which require the storage of the watermark, original signal or several additional security keys, this scheme proposes a much more efficient algorithm in the sense that except the assigned zero locations, stage number, frame size and zero tree root locations, no extra information need to be stored. The root locations matrix is a binary one and the larger the number $L$ of stages used in the wavelet decomposition, the smaller it gets in size. Even if the stage number, the frame size, and the root location matrices somehow became available to a pirate, it is still impossible to predict the security keys from the marked signal without accessing to assigned zero locations in the filter banks.

One improvement over our previously reported audio watermarking technique is that, since the zero tree coefficients of the wavelet decomposed signal are replaced with a chosen embedded intensity during encoding, the method here is more robust with respect to assigned zero locations in the filter banks. We continue to explore more robust watermarking schemes both for audio and image signals.

\section{References}

[1] M. Akbaș, Zero assigned filter banks and wavelets, Master's Thesis, Department of Electrical and Electronics Engineering, Bilkent University, 2001.

[2] P. Bao, X. Ma, Image adaptive watermarking using wavelet domain singular value decomposition, IEEE Transactions on Circuits and Systems for Video Technology 15 (1) (2005) 96-102.

[3] M.U. Çelik, G. Sharma, A.M. Tekalp, Collusion-resilient fingerprinting using random prewarping, in: Proceedings. 2003 International Conference on Image Processing, 2003. ICIP 2003, vol. 1, 2003.

[4] I.J. Cox, J. Kilian, F.T. Leighton, T. Shamoon, Secure spread spectrum watermarking for multimedia, IEEE Transactions on Image Processing 6 (12) (1997) 1673-1687.

[5] I. Daubechies, Ten Lectures on Wavelets, in: CBMS-NSF Regional Conference Series in Applied Mathematics, vol. 61, no. 4, Society for Industrial and Applied Mathematics (SIAM), Philadelphia, PA, 1992.

[6] M.S. Hsieh, D.C. Tseng, Y.H. Huang, Hiding digital watermarks using multiresolution wavelet transform, IEEE Transactions on Industrial Electronics 48 (5) (2001) 875-882.

[7] H. Inoue, A. Miyazaki, T. Katsura, An image watermarking method based on the wavelet transform, in: Proceedings. 1999 International Conference on Image Processing, 1999. ICIP 99, vol. 1, 1999.

[8] H. Inoue, A. Miyazaki, A. Yamamoto, T. Katsura, A digital watermark based on the wavelet transform and its robustness on image compression, in: Proceedings. 1998 International Conference on Image Processing, 1998. ICIP 98, vol. 2, 1998.

[9] X. Li, M. Zhang, R. Zhang, A new adaptive audio watermarking algorithm, in: Fifth World Congress on Intelligent Control and Automation, 2004. WCICA 2004, vol. 5, 2004

[10] M.K. Mıhçak, R. Venkatesan, M.H. Jakubowski, Blind image watermarking via derivation and quantization of robust semi-global statistics, preprint, 2002.

[11] A. Miyazaki, On the evaluation of wavelet filter banks for waveletbased image watermarking, in: Proceedings of the 3rd International Symposium on Image and Signal Processing and Analysis, 2003. ISPA 2003, vol. 2, 2003.

[12] H.I.A. Miyazaki, T. Katsura, A digital watermark for images using the wavelet transform, Integrated Computer-Aided Engineering 7 (2000) 105-115.

[13] K. Ratakonda, R. Dugad, N. Ahuja, Digital image watermarking: issues in resolving rightful ownership, in: Proceedings. 1998 International Conference on Image Processing, 1998. ICIP 98, vol. 2, 1998.

[14] J.M. Shapiro, D.S.R. Center, N.J. Princeton, Embedded image coding using zerotrees of wavelet coefficients, IEEE Transactions on Signal Processing 41 (12) (1993) 3445-3462 (see also IEEE Transactions on Acoustics, Speech, and Signal Processing).

[15] M.D. Swanson, M. Kobayashi, A.H. Tewfik, C. Inc, M.N. Minneapolis, Multimedia data-embedding and watermarking technologies, Proceedings of the IEEE 86 (6) (1998) 1064-1087.

[16] M.D. Swanson, B. Zhu, A.H. Tewfik, L. Boney, Robust audio watermarking using perceptual masking, Signal Processing 66 (3) (1998) 337-355.

[17] P.P. Vaidyanathan, Multirate Systems and Filter Banks, PrenticeHall, Inc., Upper Saddle River, NJ, USA, 1993.

[18] S. Voloshynovskiy, S. Pereira, T. Pun, J.J. Eggers, J.K. Su, Attacks on digital watermarks: classification, estimation based attacks, and benchmarks, IEEE Communications Magazine 39 (8) (2001) $118-126$

[19] G.K. Wallace, The JPEG still picture compression standard, IEEE Transactions on Consumer Electronics 38 (1) (1992)

[20] Y. Wang, J.F. Doherty, R.E. Van Dyck, A wavelet-based watermarking algorithm for ownership verification of digital images, IEEE Transactions on Image Processing 11 (2) (2002) 77-88.

[21] X.G. Xia, C.G. Boncelet, G.R. Arce, A multiresolution watermark for digital images, in: Proceedings of the IEEE International Conference on Image Processing 1997, ICIP, vol. 97, pp. 548-551.

[22] Y. Yardımcı, A.E. Çetin, R. Ansari, Data hiding in speech using phase coding, in: Fifth European Conference on Speech Communication and Technology. ISCA, 1997.

[23] Z. Yücel, A.B. Özgüler, An audio watermarking algorithm via zero assigned filter banks, in: European Signal Processing Conference EUSIPCO 2005, 2005.

[24] Z. Yücel, A.B. Özgüler, An image watermarking algorithm via zero assigned filter banks, in: Proceedings of the Fifth IEEE International Symposium on Signal Processing and Information Technology, vol. 1, 2005, pp. 363-368. 